\title{
Kants Beziehungen zur Psychologie und Psyehiatrie.
}

\author{
Entwurf einer Arbeit von \\ $\dagger$ Dr. Fritz Taubert, \\ weiland Oberarzt in Lauenburg i. Pomm. 1).
}

(Eingegangen am 18. März 1914.)

Die Möglichkeit einer experimentellen Psychologie wird von Kant in Frage gestellt (Metaphysische Anfangsgründe der Naturwissenschaft. Vorrede). Heute haben wir eine empirische Psychologie, eine mit naturwissenschaftlichen Methoden arbeitende Geisteswissenschaft, eine Experimental-Psychologie, Psychophysik.

$\mathrm{Zu}$ Kants Zeiten war die Psychologie vorwiegend eine rationale, ein Teil der metaphysischen Philosophie. Kant selbst hat dieser den Garaus gemacht in den Paralogismen der rationalen Psychologie und transzendentalen Dialektik. Zu Kants Zeiten erst begann eine empirische Psychologie.

Kants Einteilung der Geisteskrankheiten ist eine rein rationale, völlig in den psychologischen Anschauungen seiner Zeit befangene. Es gab ja noch keine klinische Psychiatrie, weder eine symptomatische noch nach dem Verlauf. Kant hat wahrscheinlich Geisteskranke nicht zu beobachten und zu studieren Gelegenheit gehabt. Wie waren die Verhältnisse damals in Königsberg?...

Kant teilt ganz systematisch die Geisteskrankheiten ein nach den einzelnen Seelen- und Geistesvermögen. Viel später hat z. B. noch Schopenhauer alle geistigen Störungen aus ei nem Prinzip, aus einer Störung des Gedächtnisses erklärt (Schauspieler). -

Immerhin hat Kant über Ätiologie, Erblichkeit, psychische und körperliche Ursachen der Geisteskrankheit, über Behandlung vereinzelte zerstreute Bemerkungen abgegeben, die außerordentlich modern anmuten, und in denen er seiner Zeit weit vorausgeeilt ist. Er hat sogar, was wenig bekannt sein dürfte, ein Schema der Halluzinationen gegeben: das dürfte wohl überhaupt der erste derartige Versuch sein.

Es verlohnt sich deshalb eine Zusammenstellung und kritische Besprechung dieser Äußerungen, die ein großes historisches Interesse beanspruchen können. Sie sind außer in der Anthropologie meist in

1) Das hinterlassene Manuskript ist von Herrn P. Fischer, Anstaltsgeistlicher in Lauenburg, freundlichst entziffert, aber nicht geändert worden. 
den leider sehr wenig bekannten vorkritischen Schriften Kants enthalten. Kant hat sogar den Krankheiten des Kopfes einen eigenen Aufsatz gewidmet, von dem $\mathrm{Ku}$ u o Fischer sagt, daß er einen seiner launigsten und lebendigsten Aufsätze darstellt. Dieser Aufsatz ist eine Gelegenheitsarbeit. Sie verdankt ihren Ursprung dem Auftreten eines halbverrückten Schwärmers, eines Naturmenschen, der in Begleitung eines siebenjährigen Knaben und einer Herde von Kühen, Schafen und Hunden in der Umgebung von Königsberg auftrat. In jenen Tagen erregte dieser halluzinierende, offenbar geisteskranke Naturschwärmer und noch mehr der ",kleine Wilde" das lebhafteste Interesse des unter dem frischen Eindruck der Ro ussea u schen Erziehungsideen stehenden gebildeten Publikums. Auch Kant hat sich den ",begeisterten Faunus" und seinen Buben angeschaut und angehört und sich in den Königsberger gelehrten und politischen Zeitungen darüber geäußert, später auch anonym den in Frage stehenden Aufsatz geliefert.

„Versuch über die Krankheiten des Kopfes." Dieser Aufsatz stellt sich dar als eine ,kleine Neomosaik der Gebrechen des Kopfes von der Lähmung desselben an in der Blödsinnigkeit bis zu dessen Verzückungen in der Tollheit". Er will dabei die Methode der Ärzte nachahmen, welche glauben, ihrem Patienten sehr viel genützt zu haben, wenn sie seiner Krankheit einen Namen geben $\left.{ }^{1}\right)$. - Vorher erörtert Ka nt mit scharfsinniger Begriffsbestimmung die milderen Grade, die, wie wir heute sagen würden, in der Breite des Physiologischen liegen, der Dummköpfigkeit bis zur Narrheit. Diese oft recht feinsinnigen Bemerkungen müssen wir hier übergehen. Wir greifen nur das heraus, was direkt psychiatrisches Interesse hat.

Während diese unsre Verachtung und Hohn herausfordern, verdienen diejenigen Gebrechen des Kopfes, welche die freie bürgerliche Gemeinschaft aufheben und obrigkeitliche Vorsorge und Verfügungen beanspruchen, gemeiniglich unser Mitleid. Kant teilt diese Gebrechen in solche der Ohnmacht und der Verkehrtheit. Der erste, der Blödsinnige, befindet sich in einer großen Ohnmacht des Gedächtnisses und der Vernunft, gemeiniglich auch sogar der sinnlichen Empfindungen. Diese Gebrechen sind unheilbar und lassen den Unglücklichen niemals aus dem Stande der Kindheit herausgehen, und sind zu bekannt, um ausführlich besprochen zu werden.

Die Gebrechen des gestörten Kopfes dagegen werden von Kant ausführlicher abgehandelt. Sie sind so vielerlei, als Gemütsfähigkeiten sind. Wenn wir verkehrt empfinden, so ist unser Geist verrückt. Wenn wir verkehrt urteilen, und sich der Irrtum unaufhörlich festsetzt, heißt der Irrtum Wahnsinn. Wenn wir verkehrt schließen und auf Unmöglichkeiten spekulieren, wird der Wahnsinn zum Wahnwitz. Aus diesen

1) Wie heute noch. 
Gruppen, ihren verschiedenen Graden, deren Vereinbarungen untereinander, vor allem aus ,,ihrer Einpfropfung auf mächtige Leidenschaften" vermeint nun Kant alle übrigen Erscheinungen des kranken Gehirns beschreiben zu können. Wenn die Halluzinationen oder Illusionen, wie sie auch der Gesunde im Traume hat, auch im Wachen aus irgendeiner krankhaften Ursache des Gehirns, bei guter gesunder Vernunft, für wirkliche Empfindungen gehalten werden, so ist Verzückung vorhanden. Die Verrïcktheit ist ein Traum im Wachen. Sind die Empfindungen nicht völlig chimärisch, sondern werden wirkliche Empfindungen übertrieben, so entsteht die phantastische Gemütsbeschaffenheit, die leicht in Verrücktheit übergehen kann. Z. B. die Hypochondrie, die Schwermut, die extatische Liebe. Von den Hypochondristen entwirft Kant folgendes Bild:......

Kant ist also geneigt, in der leidenschaftlichen Liebe einen gelinden Grad gestörter Geistestätigkeit zu erblicken, wie wir heute sagen würden, eine physiologische Paranoia. Von dieser phantastischen Gemiitsbeschaffenheit trennt Kant als gesund den Enthusiasmus ab, der in starkem Grade von moralischen Empfindungen als Grundsitz ersetzt wird, dem gemeinen Verstande aber leicht als phantastisch erscheint. Und doch ist niemals ohne Enthusiasmus etwas Großes in der Welt geschehen. Ganz anders der Fanatiker. Besonders, wenn dessen Schwärmerei mit der Möglichkeit der Erfahrung im Widerspruch steht. Der Fanatiker ist geisteskrank, wenn er sich einbildet, ein Auserwählter des Himmels zu sein. Solche Fanatiker sind gefährlich, weil sie ansteckend wirken können und dann epidemische Geisteskrankheiten verursachen. - Es gibt auch Phantasten des Erinnerungsvermögens.

Gibt Kant an dieser Stelle nun eine ganz allgemeine Erklärung über die Entstehung chimärischer Empfindungen, so enthält dafür das 3. Hauptstücks I. Teil die Träume eines Geistersehers, erläutert durch Träume der Metaphysik, eine eingehende Theorie der Halluzinationen. Es mag hier gleich hervorgehoben werden: Kant gebraucht hier und an andern Stellen niemals den Ausdruck Halluzination, wo er von chimärischen Empfindungen, Träumen der Empfindung usw. redet. $\mathrm{Da} B \mathrm{ihm}$ aber dieser Ausdruck nicht unbekannt ist, beweist folgende Stelle in der Anthropologie $\$ 77$ : ,Es ist so wie das Fluchen, wenn man beim Gehen an einen freiliegenden Straßenstein (mit dem großen Zeh, davon das Wort hallucinatio hergenommen) stoßßt, vielmehr ein Ausdruck des Zornes'.... Halluzinatio gebraucht Kant also im Sinne einer lauten, tobsüchtigen Erregung. (Über diese falsche Ableitung siehe auch A. Bezzenberger, Zur Erinnerung an I. Kant, Abhandl. der Universität Könisgberg 1904 S. 262.)

In den Träumen eines Geistersehers usw. beschäftigt sich K a nt mit Swedenborg. Dieser war bekanntlich vor seiner mystischen oder ma- 
gischen Periode ein um den Bergbau verdienter Mann, hatte sich durch mathematische und physikalische Schriften, durch mechanische Erfindungen bekannt gemacht. Er war schon 55 Jahre alt, als er die ersten Visionen (Christus-Erscheinungen u. dgl.) gehabt haben wollte. Fr bildete Sekten und war damals Gegenstand allgemeinen Interesses. Die ,Arcana coelestia', bilden ein hervorragendes Stïck der pathologischpsychischen Literatur. Kant erkannte ihn sehr richtig als einen Geisteskranken.

Indem er die Träume der Empfindung mit den Träumen der Vernunft - worunter er die dogmatische Metaphysik (die rationale Theologie, Kosmologie, Psychologie) der Leibnitz - Wolfschen Schule verstand - in Parallele setzte, entstanden seine geistvollen Doppelfolgerungen, ein wichtiges Dokument in dem philosophischen Entwichlungsgange Kants: er ließ die Metaphysik im Lichte der Visionäre ererscheinen und indem er diese durch jene erläuterte, traf er mit dem Pfeil seines Spottes das doppelte Ziel.... (K u no Fischer.)

Den Träumen der Vernunft setzte Kant die Träume der Empfindung zur Seite. UTmkehrung des Satzes von Aristoteles (er stammt in Wirklichkeit von Heraklit). Von dem wahren Träumer unterscheidet Kant scharf den Halluzinanten. Nicht die Lebhaftigkeit der sinnlichen Empfindung ist es auch schon nach Kant, die ein sicheres Kennzeichen liefert für die Beurteilung der Realität eines Bewußtseinszustandes, sondern daß sie in bestimmte Stellen des Raumes projiziert wird, das Erinnerungsbild dagegen nicht:

„Wir finden aber . . in der Empfindung auch ihren Ort mitbegriffen." - Es ist das Fehlen der inneren Beziehungen der Vorstellungen zu dem gesamten wirklichen Wahrnehmungsfeld. „Nun verlange ich aber mir einzuräumen - - Wenn ich also setzte..."

Kant geht von den ideae materiales des Descartes aus. Dieser Betrug kann einen jeden äußern Sinn betreffen, denn von jeglichem haben wir kopierte Bilder... Vergleich mit den Doppelbildern bei Betrunkenen!...

Kant gibt also eine zentrale Theorie der Halluzinationen. Es handelt sich um eine Erkrankung der ideae immateriales, derjenigen Organe des Gehirns, die mit einiger Phantasie harmonisch arbeiten mit der Stelle kopierter Bilder. (Also eine zentrale Erkrankung am Erinnerungsfelde der Sinnessphären.) Normalerweise schneiden sich die Richtungslinien der Bewegungen bei den Phantasien innerhalb des Gehirns, bei den Empfindungen außerhalb des Focus imaginarius (in der Wirklichkeit Zerstreuungspunkt, in der Vorstellung der Sammlungspunkt der Direktionslinien, nach welchen die Empfindung eingedrückt wird). Der focus imaginarius, darin das Objekt vorgestellt wird, liegt bei den klaren Empfindungen des Wachens außer mir, der von den Phantasien 
in mir. Die Seele versetzt das empfindende Objekt dahin in ihrer Vorstellung, wo die verschiedenen Richtungslinien des Eindrucks, die dasselbe gemacht hat, wenn sie fortgezogen werden, zusammentreffen. Dieser focus imaginarius liegt außerhalb des Gehirns bei den wirklichen Empfindungen, bei den Sinnestäuschungen im Gehirn selbst.... ..... (T. deutet ein Schema in der Zeichnung an.)

Soemmering (Sitz der Seele) verdient unser Interesse namentlich auch deshalb, weil Kant sich über seine Hypothese etwas ausführlicher äußert. Soem mering ging wieder auf die Gehirnhöhlen zurück und betrachtet die Flüssigkeit, die in diesen Höhlen enthalten ist, als den Träger der seelischen Funktionen. Auf die Bitte um eine Meinungsäußerung antwortet ihm Kant im Jahre 1796 sehr liebenswürdig, aber schließlich doch ablehnend. Kant war selbstverständlich überzeugt, daß das Gehirn der Sitz der seelischen Funktionen sei. Er bezeichnet es direkt als „Sitz des Verstandes“. Geisteskrankheiten sind für ihn schon - ebenso wie heute für uns - Gehirnkrankheiten. Dabei hebt er aber - für seine Zeit gewiß mit Re ht - hervor, daß es an empirischen Daten über den Ort der seelischen Prozesse doch noch völlig fehle. Er glaubt, daß die Aufgabe, den Sitz der Seele zu suchen, auf eine unmögliche Größe führe. - ......... Haeser sagt: Wesentlichen Vorschub leistete dem Aufschwung der Psychiatrie die von Kant angeregte philosophische Bewegung, an welcher sich nicht wenige hervorragende Ärzte beteiligten.

Von den Ärzten, welche sich zu den Lehren Kants bekannten, genügt es, seine Freunde Markus Herz, Jos. Benj. Erhardt, Arzt zu Berlin, anzuführen. (Denwürdigk. des Philosophen und Arztes J. B. Erhard, herausg. von Varnhagen, Stuttgart und Tübingen 1836.) Bohn, Ưber Kants Beziehungen zur Medizin. Königsberg 1873.

Ha eser: Die Lehren Kants äußerten, wie mit Recht gesagt worden ist, in Deutschland einen beinahe zauberischen Einfluß auf alle Wissenschaften, indem sie einen philosophischen Untersuchungsgeist rege machten, dessen man das Zeitalter nicht für fähig gehalten hätte. Kant stand wie Descartes und Leibnitz auf der Höhe der Naturwissenschaften seiner Zeit....... 